\section{Zu der Arbeit von Sorges et al. „Sofortdiagnose und Soforttherapie durch den zuerst eintreffenden Arzt", Intensivmed 36:727-736 (1999)}

Mit Interesse habe ich Ihren Beitrag zur Sofortdiagnose und Soforttherapie durch den zuerst eintreffenden Arzt in der Zeitschrift ,Intensivmedizin" gelesen. Ich nehme an, dass es sich hierbei um den akuten Myokardinfarkt handelt.

In Tabelle 2 erwähnen Sie die Gabe von Sauerstoff (auch Seite 732). Es ist natürlich bekannt, dass beim akuten Infarkt eine Hypoxämie vorliegt. Es gibt jedoch nur wenige Studien, die einen Effekt der Sauerstoffgabe auf das Herz und auf die Überlebensrate nachweisen. Dem stehen Untersuchungen gegenüber, dass bei höherer Konzentration von Sauerstoff negative Auswirkungen auf das Herz (Steigerung des peripheren Widerstandes, Einengung der Koronargefäße) auftreten können. Die Sauerstoffgabe ist natürlich eine beliebte Maßnahme, deren Wirkung jedoch kaum und unzureichend untersucht wird. Meines Erachtens be-

Prof. Dr. med. H. Löllgen (

Medizinische Klinik I

Kardiologie, Pneumologie

Klinikum Remscheid

Postfach 160180

D-42830 Remscheid steht eine Indikation dann, wenn in der Klinik auch eine Hypoxämie nachgewiesen wurde. Dann sollte auf jeden Fall eine Sauerstoffgabe, aber mit niedriger Dosierung, erfolgen, wobei 2-3 Liter pro Minute ausreichend sind. Die bekannten Nachteile lokaler Art durch die Sauerstoffgabe sollten nur am Rande erwähnt werden und stellen für viele Patienten eine erhebliche Beeinträchtigung dar. Präklinisch sollte bei Infarktverdacht eine Sauerstoffgabe nicht über 2-31/min erfolgen.

Weiterhin erwähnen sie die Gabe von $500 \mathrm{mg}$ Acetylsalicylsäure i.v. als Erstmaßnahme. Es kann kein Zweifel daran bestehen, dass die Aspiringabe als gesichert gilt. Eine von uns durchgeführte Literaturrecherche ergab aber, dass es keine Untersuchungen gibt, die eine i.v.Gabe von Aspirin insbesondere in der hohen Dosierung beim akuten Myokardinfarkt rechtfertigt oder sichert. Ich gebe natürlich zu, dass ein Notarzt nur darauf geschult wird, irgendetwas i.v. zu spritzen und keine Tablette zu verabreichen. Dennoch muss nach der derzeitigen Datenlage aller Studien die orale Gabe von Aspirin $100 \mathrm{mg}$ als ausreichend und gesichert gelten.

Bei der Antiarrhythmikatherapie wird die zurückhaltende Gabe von Lidocain mit Recht erwähnt. Meines Erachtens bedürfte es aber zumindest einer weiteren Erwähnung, dass bei Kammertachykardien Ajmalin wirksamer ist und bei schwierigen Situationen auch die Gabe von Amiodaron.

Bei der Gabe von Betarezeptorenblockern i.v. sollte erwähnt wer- den, dass nach einer i.v.-Gabe eine Monitor- oder EKG-Überwachung indiziert ist, da vor allem beim Hinterwandinfarkt häufiger als sonst eine AV-Blockierung beobachtet werden kann. Man sollte im Zweifelsfall darauf hinweisen, dass nur der erfahrene Arzt so vorgehen sollte, der weniger erfahrene wird die Betablockertherapie intravenös der Klinik oder dem Notarzt überlassen.

Schließlich wäre noch ein Hinweis wichtig, auch wenn dies nicht durch klassische Studien bisher belegt ist, dass einige der EKG-Überwachungsgeräte die Möglichkeit bieten, das EKG per Telefon an die aufnehmende Klinik zu übertragen. Dies hat den großen Vorteil, dass wenig erfahrene Notärzte sich Rat in der Klinik einholen können oder dass eine Ankündigung eines Infarktes mit größerem Nachdruck in der aufnehmenden Klinik geplant wird. Hier liegen Studien vor, dass durch präklinische EKG-Registrierung und Übertragung an die aufnehmende Klinik die Überlebensrate sogar verbessert wird. Da in manchen Städten, so auch bei uns in der Klinik, Nicht-Internisten den Notarztwagen fahren (Chirurgen, Neurologen, Pädiater), die nur unzureichend mit EKG-Beurteilungen vertraut sind, ist eine solche logistische Verbesserung sicherlich sinnvoll und erwähnenswert.

Meine Anmerkungen sollen dazu dienen, auch die präklinischen Notfallmaßnahmen auf die bisher gesicherten Studien zu begrenzen und spekulative oder übertragbare Ergebnisse mit der notwendigen Zurückhaltung und Kritik darzustellen. 